\title{
Compressive Imaging of Moving Object Based on Linear Array Sensor
}

\author{
Changjun $\mathrm{Zha},{ }^{1,2}$ Yao Li, ${ }^{2}$ Jinyao Gui, ${ }^{2}$ Huimin Duan, ${ }^{2}$ and Tailong $\mathrm{Xu}^{2}$ \\ ${ }^{1}$ Key Laboratory of Intelligent Computing \& Signal Processing, Ministry of Education, Anhui University, \\ No. 3 Feixi Road, Hefei 230039, China \\ ${ }^{2}$ Department of Electronic Information and Electrical Engineering, Hefei University, No. 99 Jinxiu Road, Hefei 230601, China
}

Correspondence should be addressed to Changjun Zha; 11586292@qq.com

Received 4 March 2016; Revised 18 June 2016; Accepted 20 June 2016

Academic Editor: Raj Senani

Copyright (c) 2016 Changjun Zha et al. This is an open access article distributed under the Creative Commons Attribution License, which permits unrestricted use, distribution, and reproduction in any medium, provided the original work is properly cited.

\begin{abstract}
Using the characteristics of a moving object, this paper presents a compressive imaging method for moving objects based on a linear array sensor. The method uses a higher sampling frequency and a traditional algorithm to recover the image through a column-bycolumn process. During the compressive sampling stage, the output values of the linear array sensor are multiplied by a coefficient that is a measurement matrix element, and then the measurement value can be acquired by adding all the multiplication values together. During the reconstruction stage, the orthogonal matching pursuit algorithm is used to recover the original image when all the measurement values are obtained. Numerical simulations and experimental results show that the proposed compressive imaging method not only effectively captures the information required from the moving object for image reconstruction but also achieves direct separation of the moving object from a static scene.
\end{abstract}

\section{Introduction}

In practical situations, objects that are observed moving across borders or uninhabited regions could be either human or animal, and there are considerable differences between their profiles $[1,2]$. Large numbers of research results have indicated that it is feasible to determine which objects are human and which are animal based on these differences, but the current research is generally concerned with the acquisition and recognition of the object profile image [38]. For image acquisition, the most common method is the use of a linear array sensor to acquire the moving object image [9-11], but the acquired image is relatively simple and cannot show the object in greater detail, meaning that it is difficult to distinguish between similar actions on different objects. Therefore, how to obtain higher-resolution images when using only a small number of sensors has become a question that is worthy of exploration.

As one of the most important research fields in compressive sensing, compressive imaging can capture a small number of measurements to be used for image reconstruction, and the most typical compressive imaging is the singlepixel camera [12-14]. To acquire compressive measurements, the camera uses a single pixel and a spatial light modulator. However, before the single-pixel camera can capture all required measurements, the scene must be in a static state or state of only slight change [15]; otherwise, the original image cannot be reconstructed. In addition, at borders or in uninhabited regions, we only care about the moving object in the monitored region and do not require a static scene. However, the reconstructed image using the singlepixel camera contains both the object and the scene, and elimination of the static scene and realization of moving object compressive sampling are subjects that require further study. To solve this problem using a combination of a linear array sensor and the theory of compressive sensing, this paper proposes a compressive imaging method for moving objects based on compressive sensing. Theoretical analyses and experimental results demonstrate the effectiveness of the proposed method. 


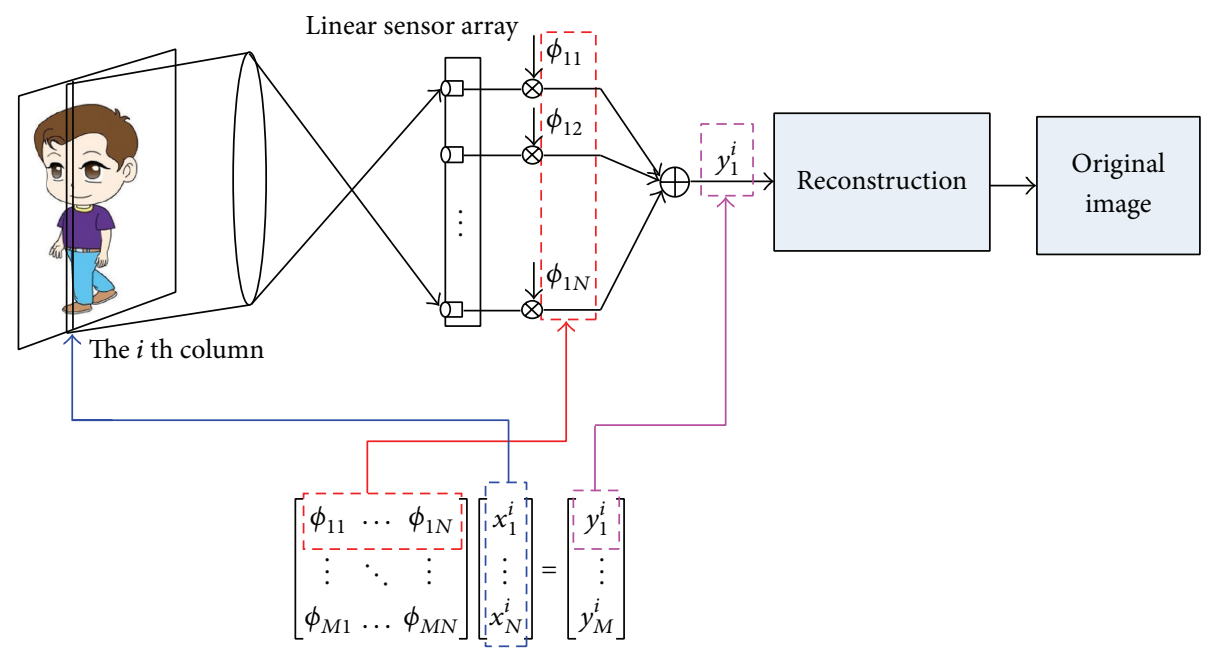

FIGURE 1: Compressive imaging system based on linear array sensor.

This paper is organized as follows. Section 2 introduces the principle of compressive sensing. Section 3 describes our proposed compressive imaging scheme in detail, and experimental results are provided in Section 4. Section 5 contains a summary of this paper and gives our conclusions.

\section{Theory of Compressive Sensing}

Consider a one-dimensional sparse signal $\mathbf{x} \in \mathbf{R}^{N}$, which can be represented as a linear combination of the columns of $\Psi \in$ $\mathbf{R}^{N \times N}$ :

$$
\mathbf{x}=\Psi \boldsymbol{\theta}
$$

If only $k(k \ll N)$ values are nonzero in the vector $\boldsymbol{\theta} \in \mathbf{R}^{N}$, then $\boldsymbol{\theta}$ is the sparse representation of $\mathbf{x}$ in the domain $\boldsymbol{\Psi}$. For the sparse signal reconstruction problem, $\boldsymbol{\theta}$ can be estimated by minimizing the $l_{1}$-norm with the measurement matrix $\Phi \in \mathbf{R}^{M \times N}(M \ll N)$ and the measurements $\mathbf{y}=\boldsymbol{\Phi} \mathbf{x} \in \mathbf{R}^{M}$ :

$$
\begin{aligned}
\widehat{\boldsymbol{\theta}}=\arg \min & \|\boldsymbol{\theta}\|_{1}, \\
\text { s.t. } & \mathbf{y}=\boldsymbol{\Phi} \boldsymbol{\Psi} \boldsymbol{\theta} .
\end{aligned}
$$

Finally, the original signal $\mathbf{x}$ can be reconstructed using the coefficient vector $\widehat{\boldsymbol{\theta}}$, which satisfies $l_{1}$-minimization $[16$, 17]; that is,

$$
\mathbf{x}=\boldsymbol{\Psi} \widehat{\boldsymbol{\theta}}
$$

In practice, when taking the effects of noise into account, (3) can be rewritten as

$$
\begin{aligned}
\hat{\boldsymbol{\theta}}=\arg \min & \|\boldsymbol{\theta}\|_{1}, \\
\text { s.t. } & \|\mathbf{y}-\boldsymbol{\Phi} \boldsymbol{\Psi} \boldsymbol{\theta}\|_{2} \leq \varepsilon,
\end{aligned}
$$

where $\varepsilon$ is the error tolerance [18-20].

For static objects, each projection measurement contains the same original information [15], but this does not apply for a moving object. We present a novel compressive imaging method to achieve compressive sampling of moving objects in the next section.

\section{Compressive Imaging System for Moving Object}

Figure 1 shows the proposed compressive imaging system for moving objects based on a linear array sensor. Unlike single-pixel cameras, the system captures the moving object image measurements through a column-by-column process. The measurement is based on the inner product of the row vector $\phi_{m}=\left[\begin{array}{llll}\phi_{m 1} & \phi_{m 2} & \cdots & \phi_{m N}\end{array}\right] \in \mathbf{R}^{N}(1 \leq m \leq M)$ of the measurement matrix $\Phi$ and the output values of the linear array sensor.

Assume here that the image of the moving object is expressed as $\mathbf{X}=\left[\mathbf{x}^{1}, \mathbf{x}^{2}, \ldots, \mathbf{x}^{N}\right] \in \mathbf{R}^{N \times N}$. We use the measurement matrix $\boldsymbol{\Phi} \in \mathbf{R}^{M \times N}(M \ll N)$ to capture compressive measurements of the $i$ th column vector $\mathbf{x}^{i}=$ $\left[\begin{array}{lll}x_{1}^{i} & \cdots & x_{N}^{i}\end{array}\right]^{T} \in \mathbf{R}^{N}$ of the image $\mathbf{X}$. Based on the theory of compressive sensing, these measurements can be expressed as $\mathbf{y}^{i}=\boldsymbol{\Phi} \mathbf{x}^{i}$; that is,

$$
\begin{aligned}
\mathbf{y}^{i} & =\left[\begin{array}{c}
y_{1}^{i} \\
\vdots \\
y_{M}^{i}
\end{array}\right]=\boldsymbol{\Phi} \mathbf{x}^{i}=\left[\begin{array}{ccc}
\phi_{11} & \cdots & \phi_{1 N} \\
\vdots & \ddots & \vdots \\
\phi_{M 1} & \cdots & \phi_{M N}
\end{array}\right]\left[\begin{array}{c}
x_{1}^{i} \\
\vdots \\
x_{N}^{i}
\end{array}\right] \\
& =\left[\begin{array}{c}
\phi_{1} \mathbf{x}^{i} \\
\vdots \\
\phi_{M} \mathbf{x}^{i}
\end{array}\right]=\left[\begin{array}{c}
\phi_{1} \\
\vdots \\
\phi_{M}
\end{array}\right] \mathbf{x}^{i},
\end{aligned}
$$

where $\mathbf{y}^{i} \in \mathbf{R}^{M}(M \ll N)$ and $\phi_{m}=\left[\begin{array}{llll}\phi_{m 1} & \phi_{m 2} & \cdots & \phi_{m N}\end{array}\right] \epsilon$ $\mathbf{R}^{N}(1 \leq m \leq M)$. During the compressive sampling stage, each row of the measurement matrix obtains a single measurement. When this measurement is obtained, the next row of the measurement matrix is used as the output coefficient of the sensor group. In this way, $M$ measurements of the $i$ th column vector of the image are obtained.

Because the object is in a state of motion, each of the rows $\phi_{m}(1 \leq m \leq M)$ of the measurement matrix samples 


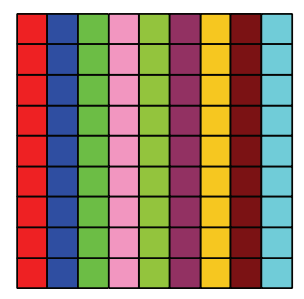

(a) Original image $\mathbf{X}$

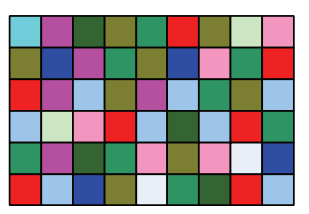

(b) Measurement matrix

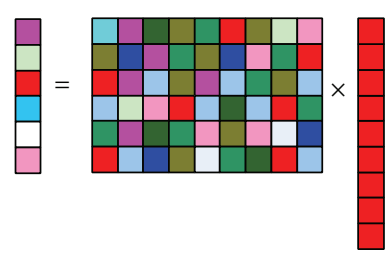

(c) Compressive sampling for static object
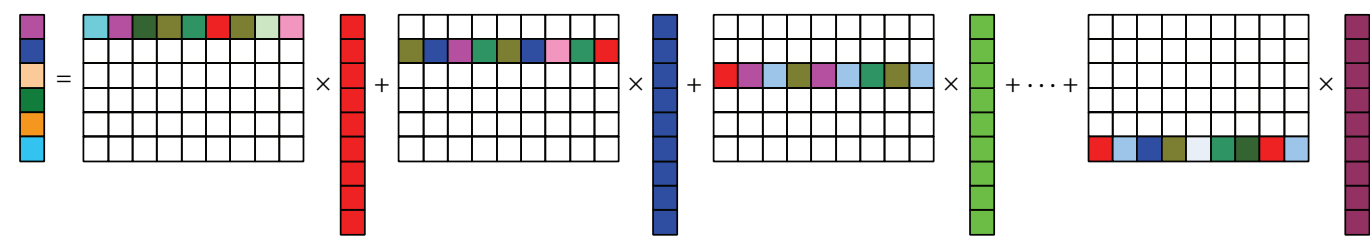

(d) Compressive sampling for moving object

FIgURE 2: Compressive sampling of static and moving states.
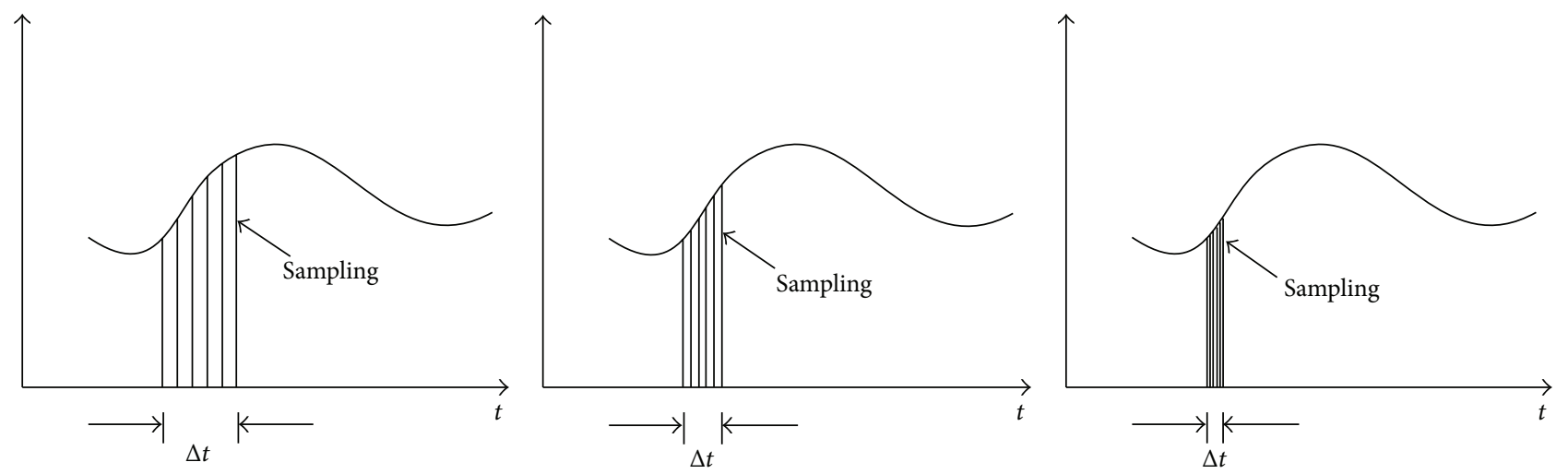

FIGURE 3: Effect of sampling frequency on the diversity of the samples.

different columns of the target image, as shown in Figure 2. Therefore, (5) should be rewritten as

$$
\mathbf{y}^{i}=\left[\begin{array}{c}
y_{1}^{i} \\
\vdots \\
y_{M}^{i}
\end{array}\right]=\left[\begin{array}{c}
\phi_{1} \mathbf{x}^{i_{1}} \\
\vdots \\
\phi_{M} \mathbf{x}^{i_{M}}
\end{array}\right]
$$

In general, the vectors $\mathbf{x}^{i_{1}}, \mathbf{x}^{i_{2}}, \ldots, \mathbf{x}^{i_{M}}(1 \leq m \leq M)$ are not equal, which means that the original image cannot be reconstructed from measurements that do not contain the same information.

In a real situation, when a person or an animal passes through the sensors' field of view, the velocity is always limited. Therefore, we could capture $M$ effective measurements $\mathbf{y}^{i}=\left[\begin{array}{lll}y_{1}^{i} & \cdots & y_{M}^{i}\end{array}\right]^{T}$ of the $i$ th column vector $\mathbf{x}^{i}=$ $\left[\begin{array}{llll}x_{1}^{i} & \cdots & x_{N}^{i}\end{array}\right]^{T}$ by using a higher sampling frequency. If we assume that the object's average velocity is $v$ and the sensors' sampling frequency is $f$, then within a short time $\Delta t$, the number of measurements $M$ can be expressed as

$$
M=f \cdot \Delta t .
$$

From (7), we see that when $M$ is fixed, $\Delta t$ becomes smaller and smaller as the sensors' sampling frequency $f$ increases, and the differences among the $M$ columns $\left\{\mathbf{x}^{i_{1}}, \mathbf{x}^{i_{2}}, \ldots, \mathbf{x}^{i_{M}}\right\}$ in the range of $\Delta t$ also become smaller, as shown in Figure 3. Assuming that the vector acquired in $\Delta t$ corresponds to the $i$ th column vector $\mathbf{x}^{i}$ of the original image, then the column vectors $\left\{\mathbf{x}^{i_{1}}, \mathbf{x}^{i_{2}}, \ldots, \mathbf{x}^{i_{M}}\right\}$ that were captured using the higher sampling frequency can be represented as

$$
\begin{gathered}
\mathbf{x}^{i_{1}}=\mathbf{x}^{i}+\boldsymbol{\varepsilon}_{1}, \\
\mathbf{x}^{i_{2}}=\mathbf{x}^{i}+\boldsymbol{\varepsilon}_{2}, \\
\vdots \\
\mathbf{x}^{i_{M}}=\mathbf{x}^{i}+\boldsymbol{\varepsilon}_{M},
\end{gathered}
$$



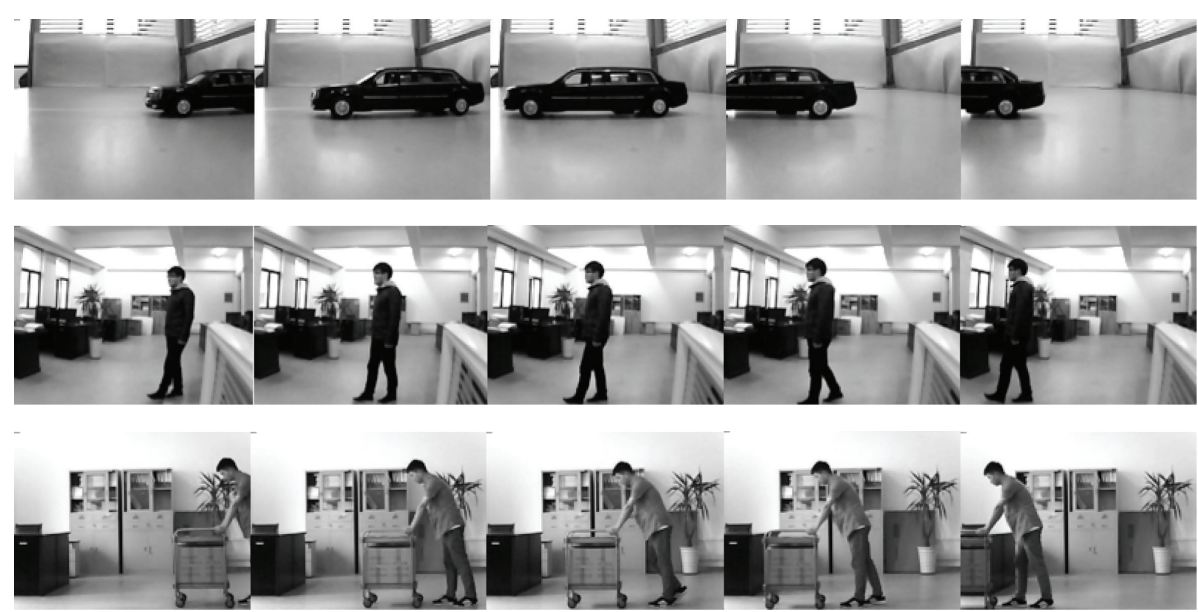

(a) Some frames in real video
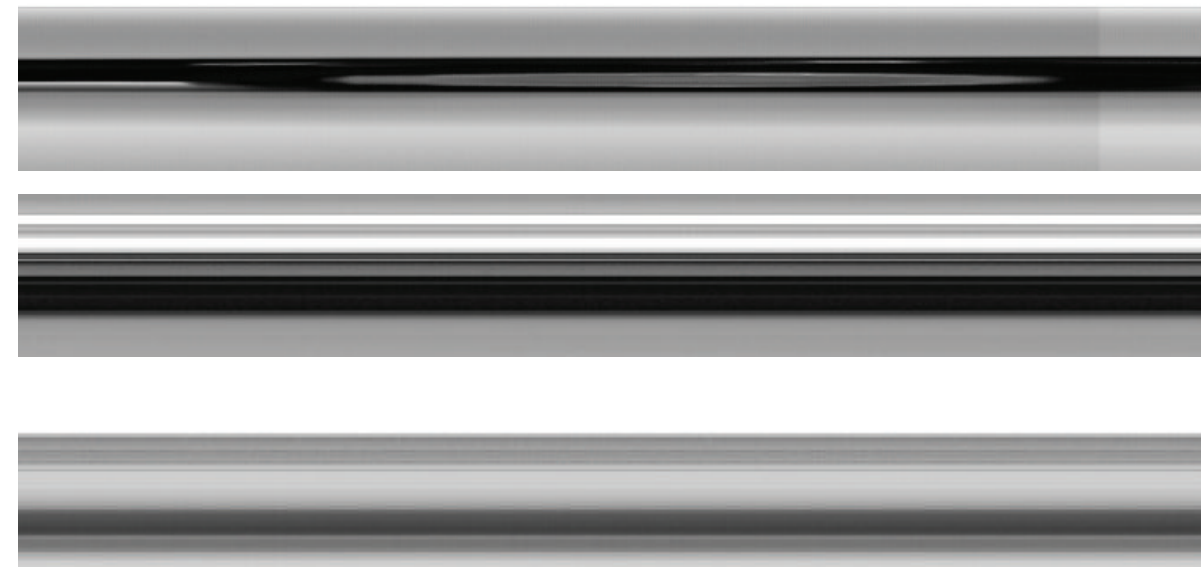

(b) Part of the data collected by a column of pixels in a camera

FIGURE 4: Real video and images.

where $\boldsymbol{\varepsilon}_{1}, \boldsymbol{\varepsilon}_{2}, \ldots, \boldsymbol{\varepsilon}_{M}$ are the error vectors. By combining (6) with (8), we obtain

$$
\begin{aligned}
\mathbf{y}^{i} & =\left[\begin{array}{c}
y_{1}^{i} \\
\vdots \\
y_{M}^{i}
\end{array}\right]=\left[\begin{array}{c}
\phi_{1} \mathbf{x}^{i_{1}} \\
\vdots \\
\phi_{M} \mathbf{x}^{i_{M}}
\end{array}\right]=\left[\begin{array}{c}
\phi_{1}\left(\mathbf{x}^{i}+\varepsilon_{1}\right) \\
\vdots \\
\phi_{M}\left(\mathbf{x}^{i}+\boldsymbol{\varepsilon}_{M}\right)
\end{array}\right] \\
& =\left[\begin{array}{c}
\phi_{1} \mathbf{x}^{i} \\
\vdots \\
\phi_{M} \mathbf{x}^{i}
\end{array}\right]+\left[\begin{array}{c}
\phi_{1} \varepsilon_{1} \\
\vdots \\
\phi_{M} \varepsilon_{M}
\end{array}\right]=\boldsymbol{\Phi} \mathbf{x}^{i}+\boldsymbol{\varepsilon},
\end{aligned}
$$

where

$$
\varepsilon=\left[\begin{array}{c}
\phi_{1} \varepsilon_{1} \\
\vdots \\
\phi_{M} \varepsilon_{M}
\end{array}\right]
$$

At this point, the $i$ th column vector $\mathbf{x}^{i}$ of the original image can be reconstructed using (4), which indicates that the entire image of the moving object can be obtained by merging all of the reconstructed columns.

For a static scene, the sensors acquire compressive measurements from the same column of the scene image. Thus, all of the columns of the reconstructed image are the same, and the reconstructed scene image is no longer the static scene that we can see.

To facilitate a clearer description, we assume that no moving objects are passing through the sensor's field of view. The reconstructed column is then expressed as $\mathbf{x}^{r}=$ $\left[\begin{array}{llll}x_{1}^{r} & x_{2}^{r} & \cdots & x_{N}^{r}\end{array}\right]^{T} \in \mathbf{R}^{N}$, and the reconstructed image can be represented as

$$
\widehat{\mathbf{X}}=\left[\begin{array}{llll}
\mathbf{x}^{r} & \mathbf{x}^{r} & \cdots & \mathbf{x}^{r}
\end{array}\right]=\left[\begin{array}{cccc}
x_{1}^{r} & x_{1}^{r} & \cdots & x_{1}^{r} \\
x_{2}^{r} & x_{2}^{r} & \cdots & x_{2}^{r} \\
\vdots & \vdots & \vdots & \vdots \\
x_{N}^{r} & x_{N}^{r} & \cdots & x_{N}^{r}
\end{array}\right],
$$

where $\widehat{\mathbf{X}} \in \mathbf{R}^{N \times N}$. From (11), we can see that the reconstructed scene is a texture image. In other words, the static 

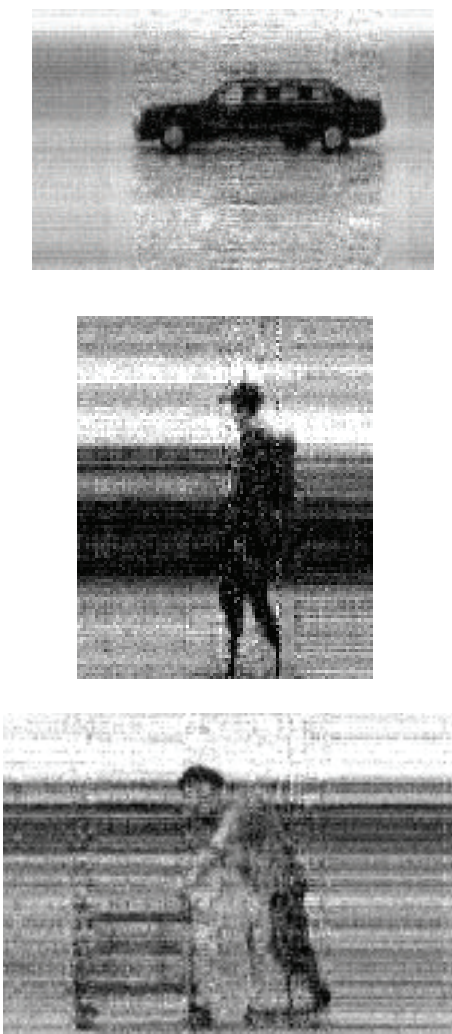

(a) $M=70$
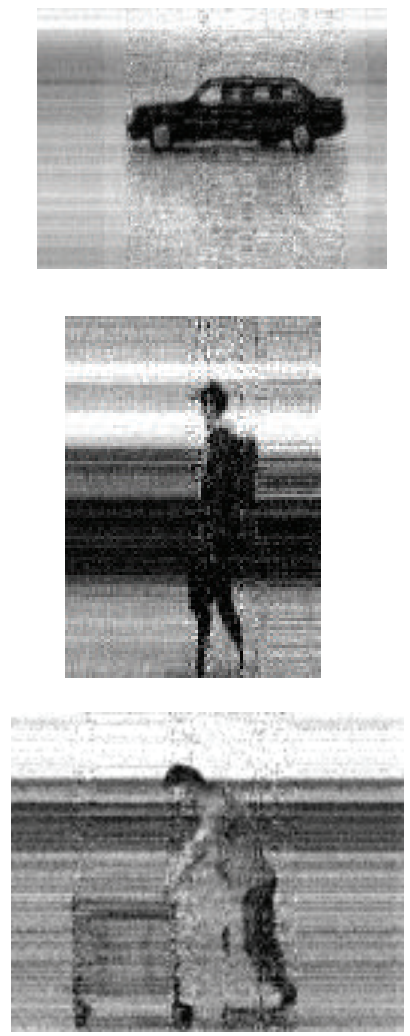

(b) $M=80$
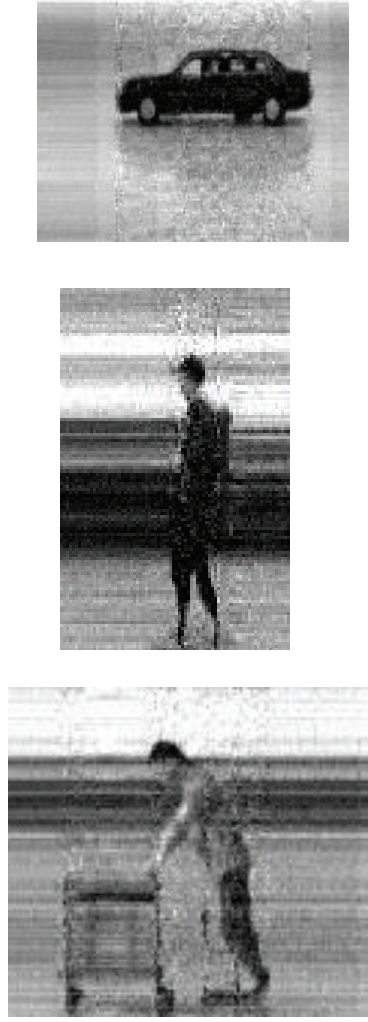

(c) $M=90$
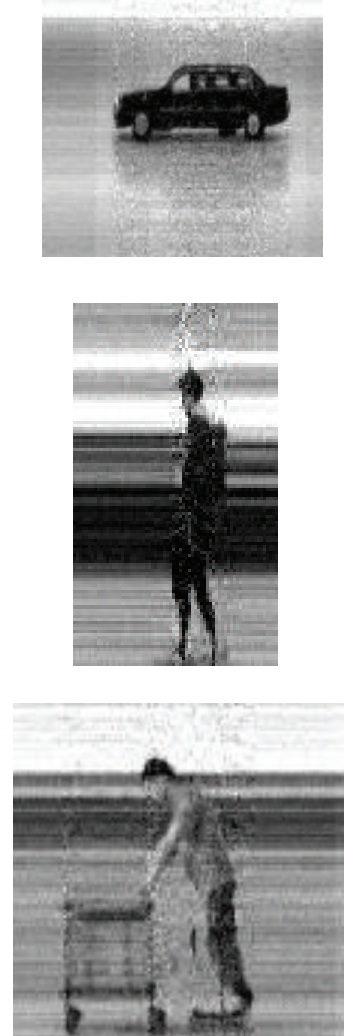

(d) $M=100$

FIgURE 5: Reconstruction of images.

scene can be eliminated using the proposed compressive imaging method.

\section{Simulation Experiment and Analysis}

To verify the validity of the proposed method, a single column pixel of an industrial camera is used to capture the data from a moving object. The number of single column pixels is 120 and the camera's frame rate is $150 \mathrm{fps}$. Some frames of the real video and a portion of the collected data under different motions and different backgrounds are shown in Figure 4.

Assuming that the sparsity level of each column in the original image is $K=30$, then the numbers of measurements $M$ are $70,80,90$, and 100 , which represent the total numbers of $58.3 \%, 66.7 \%, 75 \%$, and $83.3 \%$, respectively; we then adopt the orthogonal matching pursuit algorithm to reconstruct the original image and obtain the results shown in Figure 5.

As shown in Figure 5, the proposed compressive imaging system can reconstruct the image of a moving object. Different from Figure 4(a), the reconstructed image contains only the moving object. It is clear that the reconstruction quality improves as the number of measurements increases.

\section{Conclusions}

When a traditional single-pixel camera captures compressive measurements, the scene is required to remain in a state of rest. This paper proposed a compressive imaging method for a moving object, where the method generally adopts a higher sampling frequency to capture the measurements of the moving object through a column-by-column process. The results of simulations and real data experiments show that our method can reconstruct the image of a moving object and can separate the moving object from a static scene. Therefore, the proposed method is of practical significance for application to the monitoring of borders or uninhabited regions.

\section{Competing Interests}

The authors declare that they have no competing interests.

\section{Acknowledgments}

This work is supported by the Anhui Provincial Natural Science Foundation (no. 1508085QF118), the Hefei University Science Foundation (no. 2014xk06), the Key Projects of Research and Development Foundation of Hefei University (no. 14KY02ZD), and the Opening Foundation of Key Laboratory of Intelligent Computing \& Signal Processing (Anhui University), Ministry of Education.

\section{References}

[1] D. J. Russomanno, E. M. Carapezza, M. Yeasin, E. Jacobs, M. Smith, and S. Sorower, "Sparse detector sensor: profiling 
experiments for broad-scale classification," in Proceedings of the Unattended Ground, Sea, and Air Sensor Technologies and Applications X, vol. 6963 of Proceedings of SPIE, Orlando, Fla, USA, March 2008.

[2] C. Zha and T. Xu, "Multiple feature sample classification via joint sparse representation," Journal of Computational Information Systems, vol. 11, no. 14, pp. 5125-5133, 2015.

[3] D. J. Russomanno, S. Chari, E. L. Jacobs, and C. Halford, "Near-IR sparse detector sensor for intelligent electronic fence applications," IEEE Sensors Journal, vol. 10, no. 6, pp. 1106-1107, 2010.

[4] S. Chari, C. Halford, E. Jacobs, F. Smith, J. Brown, and D. Russomanno, "Classification of humans and animals using an infrared profiling sensor," in Unattended Ground, Sea, and Air Sensor Technologies and Applications XI, vol. 7333 of Proceedings of SPIE, Orlando, Fla, USA, April 2009.

[5] D. Russomanno, S. Chari, and C. Halford, "Sparse detector imaging sensor with two-class silhouette classification," Sensors, vol. 8, no. 12, pp. 7996-8015, 2008.

[6] R. K. Reynolds, S. Chari, and D. J. Russomanno, "Embedded real-time classifier for profiling sensors and custom detector configuration," in Ground/Air Multisensor Interoperability, Integration, and Networking for Persistent ISR II, vol. 8047 of Proceedings of SPIE, Orlando, Fla, USA, April 2011.

[7] A. Galvis and D. J. Russomanno, "Advancing profiling sensors with a wireless approach," Sensors, vol. 12, no. 12, pp. 1614416167, 2012.

[8] J. Hossen, E. L. Jacobs, and S. Chari, "Real-time classification of humans versus animals using profiling sensors and hidden Markov tree model," Optical Engineering, vol. 54, no. 7, Article ID 073102, 2015.

[9] D. J. Russomanno, S. Chari, K. Emmanuel, E. Jacobs, and C. Halford, "Testing and evaluation of profiling sensors for perimeter security," ITEA Journal, vol. 31, no. 1, pp. 121-130, 2010.

[10] R. B. Sartain, K. Aliberti, T. Alexander et al., "Long-wave infrared profile feature extractor (PFx) sensor," in Proceedings of the Unattended Ground, Sea, and Air Sensor Technologies and Applications XI, vol. 7333 of Proceedings of SPIE, Orlando, Fla, USA, April 2009.

[11] S. Chari, E. L. Jacobs, and D. Choudhary, "Pyroelectric linear array sensor for object recognition," Optical Engineering, vol. 53, no. 2, Article ID 023101, 2014.

[12] D. Takhar, J. N. Laska, M. B. Wakin et al., "A new compressive imaging camera architecture using optical-domain compression," in Computational Imaging IV, vol. 6065 of Proceedings of SPIE, San Jose, Calif, USA, January 2006.

[13] M. F. Duarte, M. A. Davenport, D. Takbar et al., "Singlepixel imaging via compressive sampling," IEEE Signal Processing Magazine, vol. 25, no. 2, pp. 83-91, 2008.

[14] P. T. Boufounos and R. G. Baraniuk, "1-Bit compressive sensing," in Proceedings of the 42nd Annual Conference on Information Sciences and Systems (CISS '08), pp. 16-21, Princeton, NJ, USA, March 2008.

[15] I. Noor and E. L. Jacobs, "Adaptive compressive sensing algorithm for video acquisition using a single-pixel camera," Journal of Electronic Imaging, vol. 22, no. 2, Article ID 021013, 2013.

[16] S. S. Chen, D. L. Donoho, and M. A. Saunders, "Atomic decomposition by basis pursuit," SIAM Journal on Scientific Computing, vol. 20, no. 1, pp. 33-61, 1998.

[17] D. L. Donoho, M. Elad, and V. N. Temlyakov, "Stable recovery of sparse overcomplete representations in the presence of noise,"
IEEE Transactions on Information Theory, vol. 52, no. 1, pp. 6-18, 2006.

[18] E. J. Candès, J. Romberg, and T. Tao, "Robust uncertainty principles: exact signal reconstruction from highly incomplete frequency information," IEEE Transactions on Information Theory, vol. 52, no. 2, pp. 489-509, 2006.

[19] E. J. Candès, J. K. Romberg, and T. Tao, "Stable signal recovery from incomplete and inaccurate measurements," Communications on Pure and Applied Mathematics, vol. 59, no. 8, pp. 12071223, 2006.

[20] J. A. Tropp and A. C. Gilbert, "Signal recovery from random measurements via orthogonal matching pursuit," IEEE Transactions on Information Theory, vol. 53, no. 12, pp. 4655-4666, 2007. 


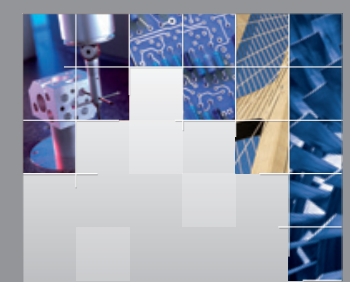

\section{Enfincering}
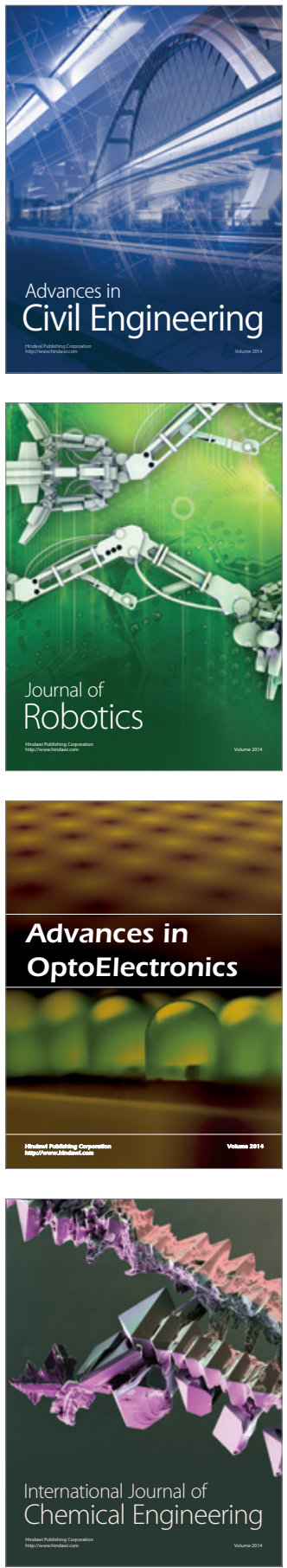

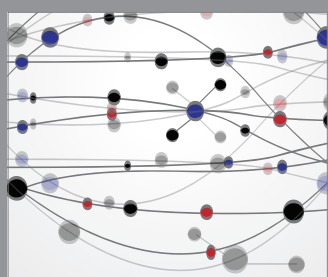

The Scientific World Journal

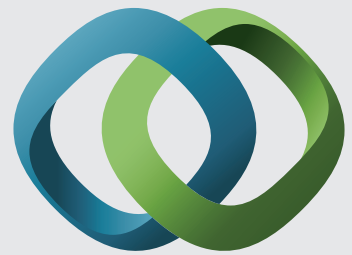

\section{Hindawi}

Submit your manuscripts at

http://www.hindawi.com
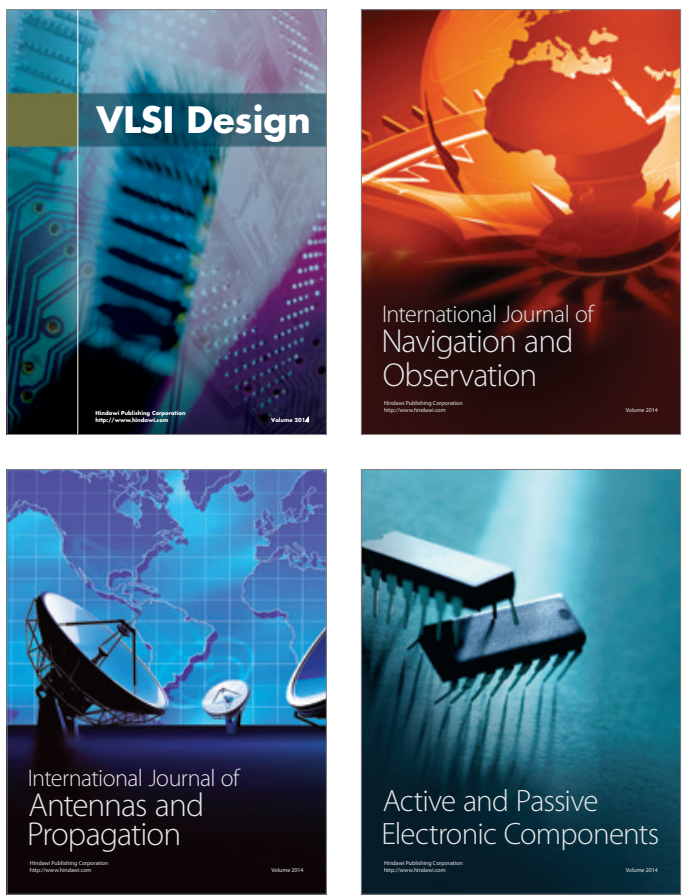
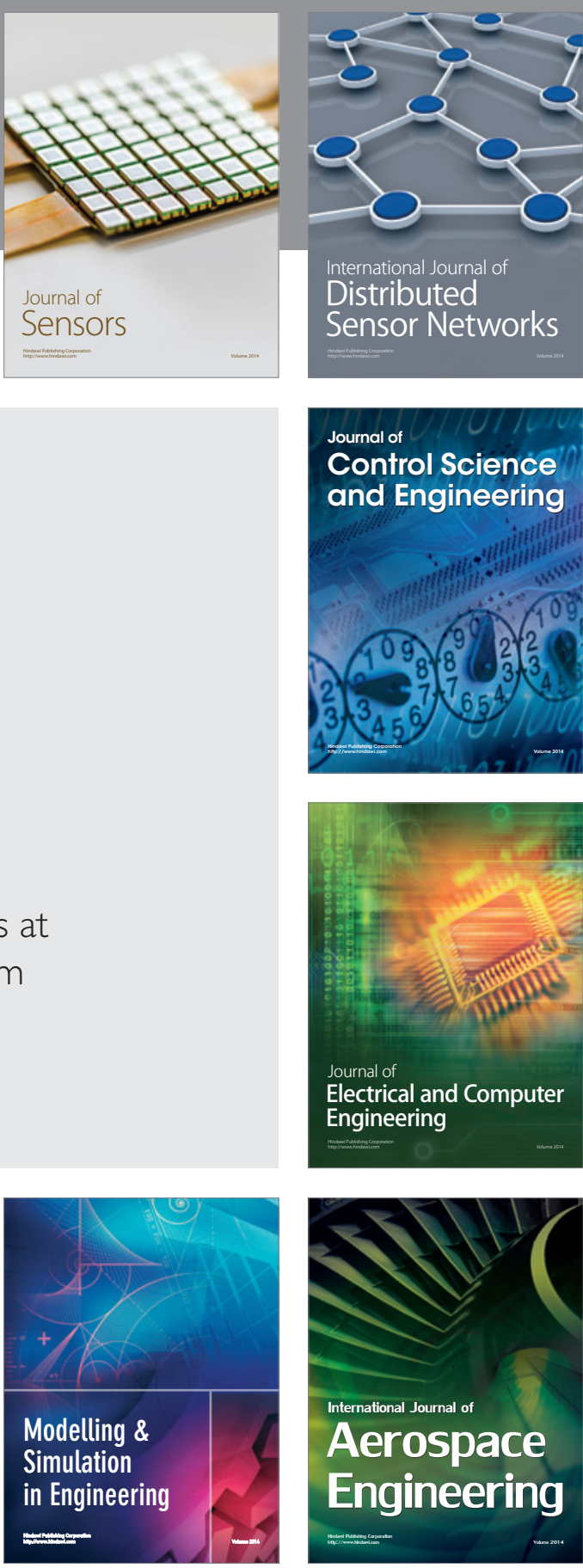

International Journal of

Distributed

Sensor Networks

Journal of

Control Science

and Engineering
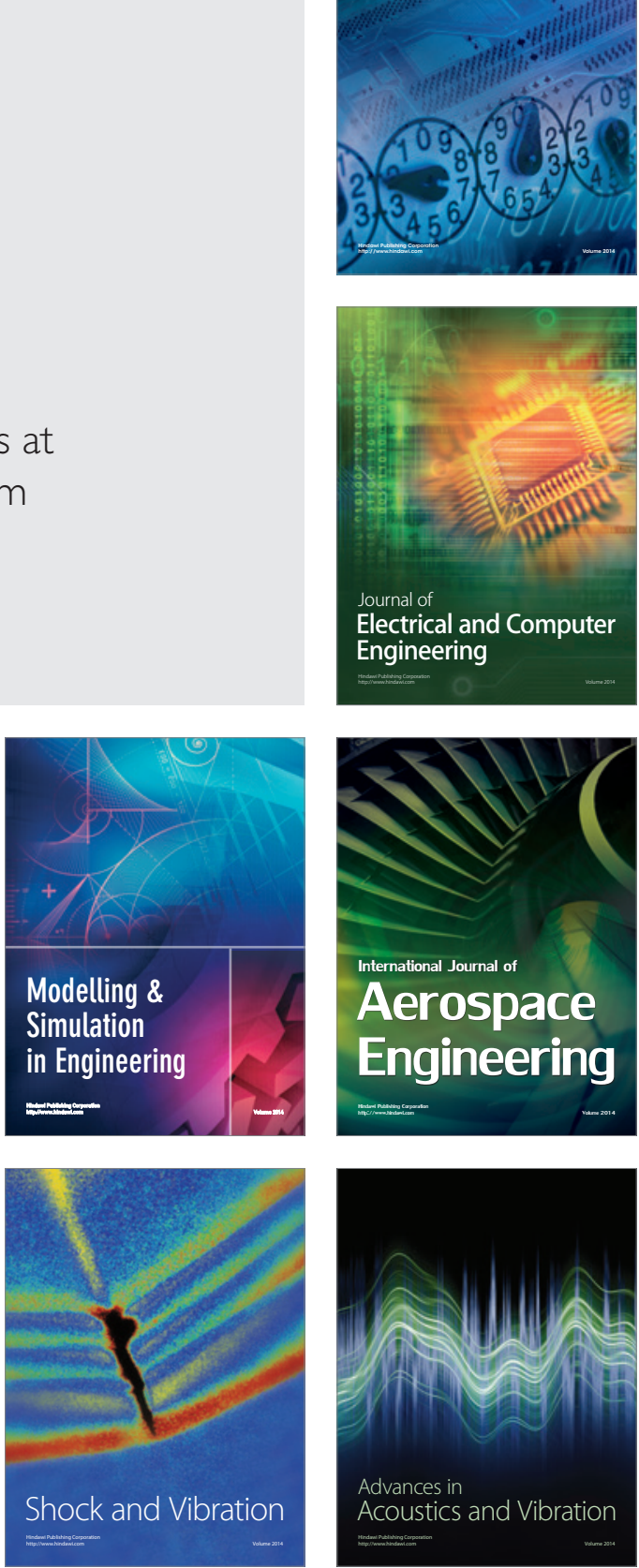